\title{
碳纳米管/聚苯胺/铁氰化镍复合膜的电化学共聚制备与电容性能
}

\author{
蔵杨郝晓刚* 王忠德张忠林刘世斌 \\ (太原理工大学化学化工学院, 太原 030024)
}

\begin{abstract}
摘要: 采用循环伏安一步共聚法在碳纳米管修饰的铂基体上制备了电活性碳纳米管/聚苯胺/铁氭化镍(CNTs/ PANI/NiHCF)复合膜. 用傅立叶变换红外(FT-IR)光谱、X 射线能谱仪 (EDS)和扫描电镜 (SEM)研究了复合膜组成 及其表面形貌, 并用循环伏安 $(\mathrm{CV})$ 、恒电流充放电和电化学阻抗(EIS)等测试了复合膜的循环稳定性与电化学容 量性能. 研究表明: CNTs/PANI/NiHCF 复合膜为三维多孔有序的网络状结构, PANI 和 NiHCF 以纳米颗粒形式 存在并沿 CNTs 均匀分布; 在电流密度为 $2 \mathrm{~mA} \cdot \mathrm{cm}^{-2}$ 时, CNTs/PANI/NiHCF 复合膜的比容量高达 $262.28 \mathrm{~F} \cdot \mathrm{g}^{-1}$, 比能量为 $29.51 \mathrm{Wh} \cdot \mathrm{kg}^{-1}$, 电流密度为 $10 \mathrm{~mA} \cdot \mathrm{cm}^{-2}$ 时比功率可达 $10228.61 \mathrm{~W} \cdot \mathrm{kg}^{-1}$; 在 2000 次循环充放电过程 中, 复合膜的电容量仅衰减 $19.92 \%$, 电荷充放电效率一直保持在 $99 \%$ 以上. CNTs/PANI/NiHCF 有机-无机杂化膜 具有良好的功率特性和快速充放电能力, 是一种优异的超级电容器材料.
\end{abstract}

关键词：超级电容器; 聚苯胺; 铁氭化锦; 碳纳米管; 复合膜; 电化学共聚; 电容性能 中图分类号: 0646

\section{Copolymerization and Capacitive Performance of Composite Carbon Nanotubes/Polyaniline/Nickel Hexacyanoferrate Films}

\author{
ZANG Yang HAO Xiao-Gang * WANG Zhong-De ZHANG Zhong-Lin LIU Shi-Bin \\ (College of Chemistry \& Chemical Engineering, Taiyuan University of Technology, Taiyuan 030024, P. R. China)
}

\begin{abstract}
Electroactive composite films of carbon nanotubes/polyaniline/nickel hexacyanoferrate (CNTs/PANI/ $\mathrm{NiHCF}$ ) were synthesized on platinum substrates modified with CNTs by a one-step co-polymerization using cyclic voltammetry. The composite films were characterized by Fourier transform infrared spectroscopy (FT-IR), energy dispersive X-ray spectroscopy (EDS), and scanning electron microscopy (SEM). Cyclic voltammetry (CV), galvanostatic charge/ discharge, and electrochemical impedance spectroscope (EIS) methods were used to study the cycling stability and the electrochemical capacitive performance of the CNTs/PANI/NiHCF films. Results showed that three-dimensional porous network composite films with uniform distributions of PANI and NiHCF nanoparticles on the CNTs were formed by this new method. The specific capacitance of the inorganic-organic hybrid films were $262.28 \mathrm{~F} \cdot \mathrm{g}^{-1}$ with a specific energy of $29.51 \mathrm{Wh} \cdot \mathrm{kg}^{-1}$ at a current density of $2 \mathrm{~mA} \cdot \mathrm{cm}^{-2}$. The specific power was $10228.61 \mathrm{~W} \cdot \mathrm{kg}^{-1}$ at a current density of $10 \mathrm{~mA} \cdot \mathrm{cm}^{-2}$. Meanwhile, CNTs/PANI/NiHCF films showed a capacity decay of only $19.92 \%$ after 2000 charge/discharge cycles and had a coulombic efficiency of over 99\%. Therefore, the composite films exhibit outstanding power performance, fast dynamics of charge transport and are excellent materials for use in supercapacitors.
\end{abstract}

Key Words: Supercapacitor; Polyaniline; Nickel hexacyanoferrate; Carbon nanotube; Composite film; Electrochemical co-polymerization; Capacitive performance

\footnotetext{
Received: August 31, 2009; Revised: November 28, 2009; Published on Web: December 31, 2009.

*Corresponding author. Email: xghao@tyut.edu.cn; Tel: +86-351-6018554.

The project was supported by the National Natural Science Foundation of China (20676089), Natural Science Foundation of Shanxi Province, China (2007011029), and Scholar Council Foundation of Shanxi Province, China (2008-32).

国家自然科学基金(20676089), 山西省自然科学基金(2007011029)和山西省回国留学基金(2008-32)资助项目
}

(C) Editorial office of Acta Physico-Chimica Sinica 
电化学超级电容器(electrochemical supercapacitor, ESC)介于传统静电电容器与化学电源之间, 是 一种利用电极/电解液界面上的双电层或在界面上 发生快速且可逆的法拉第反应来储存能量的新型储 能装置 ${ }^{[1-3]}$, 在信息技术、移动通讯、电动汽车、航天 航空和国防科技等领域具有广阔的应用前景 ${ }^{[4-6]}$.

电极材料是超级电容器的主要组成部分和研究 开发的重点, 迄今为止所研究的超级电容材料主要 包括高比表面积碳材料 ${ }^{[7-9] 、}$ 、过渡金属氧化物 $[2,6]$ 、导 电聚合物 ${ }^{[4,10-15]}$ 及其复合物 ${ }^{[3,5,16-19]}$. 多壁碳纳米管 (CNTs) 由于其具有高比表面积、低电阻和纳米多孔 形貌曾被认为是理想的超级电容器材料, 但 CNTs 仅具有双电层电容特征, 其比电容通常只有 20-80 $\mathrm{F} \cdot \mathrm{g}^{-1[5,7]}$. 过渡金属氧化物和导电聚合物的噟电容特 点使其比电容大幅增加而成为最具潜力的超级电容 器材料 ${ }^{[9,16]}$. 金属氧化物中 $\mathrm{RuO}_{2}$ 具有最高的比电容 但成本高且有毒性, 因此研究人员一直在寻求其它 环境友好低成本的替代物如 $\mathrm{MnO}_{2}{ }^{\left[{ }^{[2]}\right.} 、 \mathrm{Fe}_{3} \mathrm{O}_{4}{ }^{\left[{ }^{[6]}\right.} 、 \mathrm{SnO}_{2}{ }^{[19]}$ 等金属氧化物, 但这些材料电极的比容量都不超过 $130 \mathrm{~F} \cdot \mathrm{g}^{-1}$ 且循环稳定性较差. 导电聚合物如聚苯胺

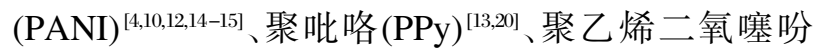
(PEDOT) $)^{[11]}$ 等由于具有良好的环境稳定性和优异的 电化学性能以及无毒、易聚合, 被认为是最具有实用 价值的超级电容器材料而日益受到人们的重视; 但 导电聚合物在脱掺杂状态下因离子迁出使体积收 缩, 且导电性下降而导致其氧化还原的可逆性和稳 定性降低 ${ }^{[2] 1}$. 为了提高导电聚合物的电容性能, 近年 来电极材料的研究重点集中在制备碳纳米管与聚合 物的复合材料或者聚合物与金属氧化物形成的有机-

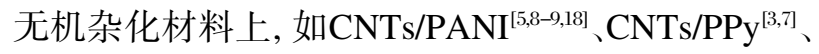

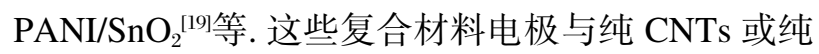
聚合物电极相比均显示了更高的电容性能, 但充放 电循环的稳定性尚有不足, 如 CNTs/PPy 复合材料 经 200 次循环电容衰减量接近 $20 \%$ 而, 而 CNTs/ PANI 复合膜 1000 次充放电循环比电容衰减了 $32 \%{ }^{[5]}$. 此外还有一类电活性材料即普鲁士蓝的过渡 金属类似物 (metal hexacyanoferrate, MHCF) 由于其 独特的化学计量关系和分子结构特征而备受关注, 如NiHCF被广泛研究用于新型电控离子交换(ESIX) 技术的膜电极材料 ${ }^{[22-24]}$, 特别是纳米管结构 $\mathrm{NiHCF}$ 具有优良的循环稳定寿命 ${ }^{[25]}$. 研究表明具有电化学 可逆特性的 MHCF 亦具备良好的电容性能 ${ }^{[26]}$, $\mathrm{CNTs} / \mathrm{MHCF}$ 纳米复合物电极用于生物传感器 ${ }^{[27-28]}$
也获得了良好效果. 最近一种新型有机-无机纳米复 合材料引起人们的高度关注, 如 Lisowska-Oleksiak 等 ${ }^{[29-30]}$ 通过两步电化学聚合获得了具有三维分布 MHCF 氧化还原活性中心的 PEDOT/MHCF 杂化 膜; Kulesza 等 ${ }^{[31-33]}$ 采用交替浸渍+电聚合方法合成 了 PyBA/PEDOT/NiHCF 以及磷钼酸 $\left(\mathrm{PMo}_{12}\right)$ 修饰的 $\mathrm{CNTs} / \mathrm{PEDOT}$ (PANI) 三维网络结构杂化膜. 由于带 正电的导电聚合物与带负电的 $\mathrm{MHCF}$ (或 $\mathrm{PMo}_{12}$ 修 饰的 CNTs)之间的静电吸引作用, 复合材料显示出 快速的离子传递能力和良好的化学稳定性, 但上述 多步合成方法相当复杂且作为 ESC 材料其循环稳 定性能尚需改进(复合膜经 500 次循环电容损失约 $15 \% \%^{[30]}$ ).

由上可知探索新的合成方法制备优良性能的新 型有机-无机复合电极材料仍然是目前 ESC 研发的 热点. 由无机过渡金属复合物和有机导电聚合物的 氧化还原作用产生的赝电容可增加碳纳米结构的比 电容, 而 NiHCF 氧化还原过程的电势范围正好与 PANI 的导电范围重合, 因而可以通过简单的一步 电化学共聚方法直接制得 PANI/NiHCF 复合膜. Kulesza 等 ${ }^{[3]}$ 曾在玻碳电极上制备了 PANI/NiHCF 复合膜, 但其形貌或微结构与单一的 PANI 膜相近 且在中性电解液中不具备电活性; Lin 等 ${ }^{[35}$ 采用分步 电化学聚合先后沉积 PANI 和 NiHCF 制得高稳定 性 CNTs/PANI/NiHCF 杂化膜用于 ESIX 过程, 但合 成步骤复杂且电化学容量偏低. 本文将采用循环伏 安一步共聚法在碳纳米管修饰的铂基体上制备电活 性 CNTs/PANI/NiHCF 复合膜, 并考察复合膜的电 容性能与循环稳定性, 以获得具有高比电容和高稳 定性能的新型结构有机-无机杂化超级电容器电极 材料.

\section{1 实验部分}

\section{1 仪器与试剂}

实验所用试剂均为分析纯或优级纯, 水为超纯 水(Millipore $18.2 \mathrm{M} \Omega \cdot \mathrm{cm}$ ), 碳纳米管由中国科学院 过程工程研究所提供(纯度 $>95 \%$, 平均直径 10-20 $\mathrm{nm})^{[36}$. 电化学测试系统均采用通过 EC-Lab 软件控 制的多通道 Princeton VMP3 恒电位仪. 用岛津 FTIR-8400 工作站对样品进行红外光谱分析. 用美 国 Kevex.Sigama 型能谱仪对样品进行 EDS 与 SEM 分析. 复合膜的质量通过 AGl35 型电子天平测量 (瑞士 Mettler-Toledo 公司, 精度 $0.01 \mathrm{mg}$ ). 


\section{2 电极预处理}

将 $1 \mathrm{~cm} \times 1 \mathrm{~cm}$ 的铂片先用氧化铝打磨成镜面, 使铂片的单面有效接触面积为 $0.5 \mathrm{~cm}^{2}$. 使用前将铂 电极放人 $1 \mathrm{~mol} \cdot \mathrm{L}^{-1} \mathrm{H}_{2} \mathrm{SO}_{4}$ 溶液中进行电化学清洗, 以 $50 \mathrm{mV} \cdot \mathrm{s}^{-1}$ 的扫描速率在 $-275-1675 \mathrm{mV}$ 之间循 环 50 次以清洁表面, 取出后用纯水冲洗晾干待用.

\section{3 复合膜的制备}

称取 $25 \mathrm{mg}$ 碳纳米管 $(\mathrm{CNTs})$ 置于 $5 \mathrm{~mL} \mathrm{~N}, \mathrm{~N}$-二 甲基甲酰胺(DMF)溶液并在超声波细胞粉碎机中超 声 $10 \mathrm{~min}$, 使 CNTs 均匀分散于 DMF 溶液. 然后用 微型移液管均匀涂在预处理过的铂片上, 室温下干 燥 $48 \mathrm{~h}$ 待用.

以 CNTs 修饰的铂片作为工作电极, 饱和甘采 电极 (SCE) 作为参比电极, 铂片作为对电极, 组成三 电极体系, 在新鲜配制的电解液 (含 $0.002 \mathrm{~mol} \cdot \mathrm{L}^{-1}$ $\mathrm{NiSO}_{4} 、 0.25 \mathrm{~mol} \cdot \mathrm{L}^{-1} \mathrm{Na}_{2} \mathrm{SO}_{4} 、 0.002 \mathrm{~mol} \cdot \mathrm{L}^{-1} \mathrm{~K}_{3} \mathrm{Fe}(\mathrm{CN})_{6}$ 、 $0.01 \mathrm{~mol} \cdot \mathrm{L}^{-1}$ 苯胺单体与 $\left.0.5 \mathrm{~mol} \cdot \mathrm{L}^{-1} \mathrm{H}_{2} \mathrm{SO}_{4}\right)$ 中进行 循环伏安一步共聚, 以 $50 \mathrm{mV} \cdot \mathrm{s}^{-1}$ 扫描速度在 $-0.2-$ $0.85 \mathrm{~V}$ 之间经25次循环扫描制得CNTs/PANI/NiHCF 复合膜. 薄膜制成后取出用纯水冲洗干净, 晾干 备用.

\section{4 复合膜性能实验}

以 $\mathrm{CNTs} / \mathrm{PANI} / \mathrm{NiHCF}$ 复合膜作工作电极, $\mathrm{SCE}$ 作参比电极, 铂片作辅助电极, 在 $0.5 \mathrm{~mol} \cdot \mathrm{L}^{-1} \mathrm{H}_{2} \mathrm{SO}_{4}+$ $0.5 \mathrm{~mol} \cdot \mathrm{L}^{-1} \mathrm{KNO}_{3}$ 混合溶液中采用循环伏安(扫描范 围为 $-0.2-0.75 \mathrm{~V}$ )、恒电流充放电(电压范围为 -0.2 $-0.7 \mathrm{~V}$ )、电化学阻抗等技术研究其电容性能.

\section{2 结果和讨论}

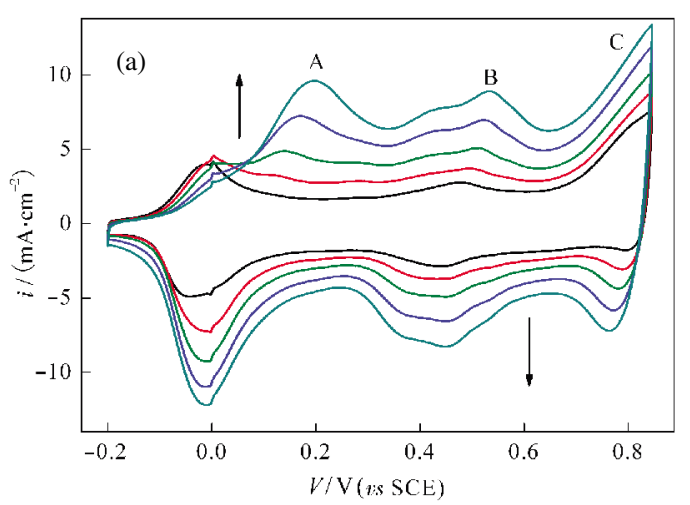

\section{$2.1 \mathrm{CNTs} / \mathrm{PANI} / \mathrm{NiHCF}$ 复合膜的共聚制备}

通过循环伏安电沉积技术可分别在导电基体上 制得 NiHCF 或 PANI 薄膜, 而 NiHCF 膜在含 $\mathrm{Na}^{+}$或 $\mathrm{K}^{+}$的碱金属溶液中进行循环扫描时氧化还原峰电位 一般出现在 $0.3-0.7 \mathrm{~V}$ 之间 ${ }^{[22-23,25]}$, 正好与 PANI 在 酸性溶液中的导电范围 $(-0.2-0.8 \mathrm{~V})^{[14]}$ 重叠, 因此 可以考虑选择适宜的混合制膜液通过循环伏安一步 共沉积方法在导电基体上直接合成 PANI/NiHCF 复 合膜. 图 1 为 $\mathrm{Pt} / \mathrm{CNTs}$ 基体在新鲜制备液中进行 $\mathrm{CNTs} / \mathrm{PANI} / \mathrm{NiHCF}$ 复合膜电沉积过程的循环伏安 曲线(a)以及与沉积次数相对应的电量增长曲线(b). 图 $\mathrm{l}$ (a) 的 CV 曲线中分别在 $0.2 / 0 \mathrm{~V}(\mathrm{~A}) 、 0.53 / 0.45 \mathrm{~V}$ (B)和 $0.85 / 0.77 \mathrm{~V}(\mathrm{C})$ 左右出现 3 对氧化还原峰, 其中 (A)和(C)分别对应着PANI由完全还原态(leucoemeraldine state) 向半氧化态(emeraldine state) 以及半氧 化态向完全氧化态(pernigraniline state)转换的峰电 位 ${ }^{[37]}$, (B) 则对应着 $\mathrm{NiHCF}$ 的氧化还原峰电位. 循环 伏安正向扫描时苯胺单体聚合得到PANI, 反向扫描 时首先将 $\mathrm{Fe}(\mathrm{CN})_{6}^{3-}$ 还原为 $\mathrm{Fe}(\mathrm{CN})_{6}^{4-}$, 再与 $\mathrm{Ni}^{2+}$ 反应沉 积得到 NiHCF薄膜 ${ }^{[35]}$. 由于半氧化态PANI带有正 电荷而NiHCF颗粒具有负电性 ${ }^{[34]}$, 两组分交替沉积 在CNTs表面并产生静电吸引, 因而可使复合膜的稳 定性显著提高. 由图可知随着循环次数的增加, 峰电 流密度随之增加, 相应的积分电荷量也在增加且与 循环次数呈线性关系, 说明薄膜在一定范围内, 能以 稳定的速度增长, 通过控制沉积次数可获得一定厚 度的复合膜, 从而控制其电化学容量的大小.

为进一步考察复合膜的一步共沉积机理及区分 膜中有机-无机组分之间的相互作用, 以Pt/CNTs为基

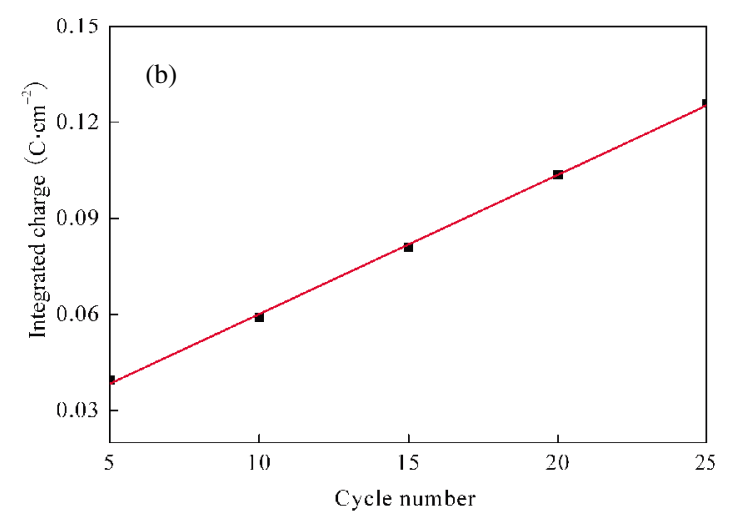

图 1 在 Pt/CNTs 基体上电沉积 CNTs/PANI/NiHCF 复合膜的表征

Fig.1 Characteristics of electrodeposition of CNTs/PANI/NiHCF films on platium electrode modified with CNTs $i$ : current density; (a) Cycle voltammograms of the film deposition process are shown every 5th cycle, (b) the integrated charge grows with deposition cycle number over all 25 cycles used. 
体在新鲜制备液中分别制备了 $\mathrm{CNTs} / \mathrm{PANI} / \mathrm{NiHCF}$ 、 $\mathrm{CNTs} / \mathrm{PANI}$ (制膜液为 $0.01 \mathrm{~mol} \cdot \mathrm{L}^{-1}$ 苯胺单体与 0.5 $\mathrm{mol} \cdot \mathrm{L}^{-1} \mathrm{H}_{2} \mathrm{SO}_{4}$ ) 与 $\mathrm{CNTs} / \mathrm{NiHCF}($ 制膜液只含 0.002 $\mathrm{mol} \cdot \mathrm{L}^{-1} \mathrm{NiSO}_{4}, 0.25 \mathrm{~mol} \cdot \mathrm{L}^{-1} \mathrm{Na}_{2} \mathrm{SO}_{4} 、 0.002 \mathrm{~mol} \cdot \mathrm{L}^{-1}$ $\left.\mathrm{K}_{3} \mathrm{Fe}(\mathrm{CN})_{6}\right)$ 三种薄膜. 各种膜在 $0.5 \mathrm{~mol} \cdot \mathrm{L}^{-1} \mathrm{H}_{2} \mathrm{SO}_{4}+$ $0.5 \mathrm{~mol} \cdot \mathrm{L}^{-1} \mathrm{KNO}_{3}$ 混合溶液中的循环伏安曲线如图 2 所示, 其中曲线 $\mathrm{d}$ 是 $\mathrm{b}$ 和 $\mathrm{c}$ 叠加而成 $(\mathrm{CNTs} / \mathrm{PANI}+$ $\mathrm{CNTs} / \mathrm{NiHCF}$ )的循环伏安曲线. 为了避免 PANI 组 分在过氧化状态下发生分解 ${ }^{[38]}$, 实验中循环扫描电 位一般控制在 $0.75 \mathrm{~V}$ 以下. 由图可见 CNTs/PANI (b)膜主要表现出由还原态向半氧化态转换的特征峰 (氧化还原峰电位 $0.15 /-0.02 \mathrm{~V}$ ), 是 PANI 在质子酸 溶液中的掺杂与去掺杂过程; 而 CNTs/NiHCF(c)薄 膜在含 $\mathrm{K}^{+}$溶液中表现出典型的双峰特征(高电位氧 化还原峰 $0.65 / 0.62 \mathrm{~V})^{[22]}$, 分别对应 $\mathrm{NiHCF}$ 中 $\mathrm{K}^{+}$的 掺人与脱出; CNTs/PANI/NiHCF(a)复合膜则包含有 PANI 和 NiHCF 两组分共有的氧化还原峰. 比较曲 线 $\mathrm{a}$ 和曲线 $\mathrm{d}$ 可知, $\mathrm{CNTs} / \mathrm{PANI} / \mathrm{NiHCF}$ 复合膜并非 $\mathrm{PANI}$ 与 $\mathrm{NiHCF}$ 两种物质的简单叠加, 复合膜中 $\mathrm{PANI}$ 与 $\mathrm{NiHCF}$ 的氧化峰电位分别向右移动了 50 和 $25 \mathrm{mV}$, 可归因于复合膜中带正电荷的PANI与带负 电荷的NiHCF组分之间存在的静电吸引作用. 此外, 一步共聚CNTs/PANI/NiHCF复合膜显示了较高的 电活性和离子交换容量, CV图的阳极峰电流密度高 达 $10 \mathrm{~mA} \cdot \mathrm{cm}^{-2}$; 而两步电沉积CNTs/PANI/NiHCF复 合膜的CV图主要呈现外层 NiHCF的电化学特征且 阳极峰电流密度不足 $1 \mathrm{~mA} \cdot \mathrm{cm}^{-2}[35]$. 图 2 中还给出 了 $\mathrm{CNTs} / \mathrm{PANI} / \mathrm{NiHCF}$ 复合膜在 $0.5 \mathrm{~mol} \cdot \mathrm{L}^{-1} \mathrm{KNO}_{3}$ 中性电解液中的 CV 曲线(e), 由图可见新型复合膜

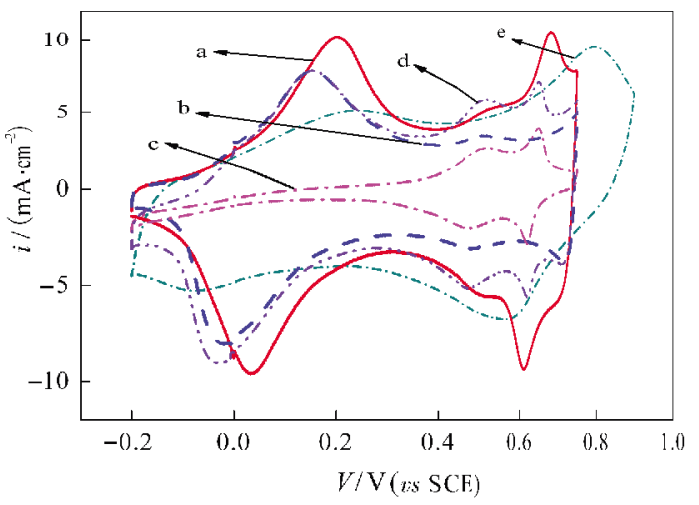

图 2 不同薄膜的循环伏安曲线

Fig.2 Voltammetric cycles of different films scan rate: $50 \mathrm{mV} \cdot \mathrm{s}^{-1}$; (a) CNTs/PANI/NiHCF, (b) CNTs/PANI, (c) $\mathrm{CNTs} / \mathrm{NiHCF}$, (d) CNTs/PANI+ CNTs/NiHCF in $0.50 \mathrm{~mol} \cdot \mathrm{L}^{-1} \mathrm{H}_{2} \mathrm{SO}_{4}+$ $0.5 \mathrm{~mol} \cdot \mathrm{L}^{-1} \mathrm{KNO}_{3}$, (e) CNTs/PANI/NiHCF in $0.5 \mathrm{~mol} \cdot \mathrm{L}^{-1} \mathrm{KNO}_{3}$
在中性电解液中也显示出良好的电活性, 而 Kulesza 等 ${ }^{[34]}$ 用一步共沉积技术制备的 PANI/NiHCF 复合膜 在中性电解液 $(\mathrm{pH}=7)$ 中并不具有电活性, 表明 $\mathrm{CNTs} / \mathrm{PANI} / \mathrm{NiHCF}$ 复合膜与 PANI/NiHCF 复合膜 应该具有完全不同的微结构特征.

\section{$2.2 \mathrm{CNTs} / \mathrm{PANI} / \mathrm{NiHCF}$ 复合膜的组成与微观形 貌分析}

图 3 为 $\mathrm{CNTs} / \mathrm{PANI} / \mathrm{NiHCF}$ 复合膜的红外光谱 图. 由图可见, $2086 \mathrm{~cm}^{-1}$ 处是 $\mathrm{NiHCF}$ 中- $\mathrm{CN}$ 一伸 缩振动 ${ }^{[25]}$, 证明有 $\mathrm{NiHCF}$ 组分存在; 而 $877 \mathrm{~cm}^{-1}$ 处 对应的是苯环上 $\mathrm{C}-\mathrm{H}$ 的面外弯曲振动, $1045 \mathrm{~cm}^{-1}$ 处对应 $\mathrm{C}-\mathrm{H}$ 的面内弯曲振动, $1272 \mathrm{~cm}^{-1}$ 处对应和 醌式有关的 $\mathrm{C}-\mathrm{N}$ 伸缩振动, 1089 与 $1627 \mathrm{~cm}^{-1}$ 处 对应醌式结构的特征吸收峰, 2927 和 $2974 \mathrm{~cm}^{-1}$ 处 对应 $\mathrm{C}-\mathrm{H}$ 的伸缩振动, $3413 \mathrm{~cm}^{-1}$ 处对应苯胺上 $\mathrm{N}-\mathrm{H}$ 的伸缩振动 ${ }^{[14,19]}$, 均为 PANI 的特征峰. 和单 一的 NiHCF 或 PANI 相比, 特征峰的位置发生了偏 移, 说明复合膜中 PANI 和 NiHCF 存在一定的相互 作用, 电子和电荷在 PANI 分子链中的离域化作用 增强, 有利于两种物质的紧密结合.

图 4 为复合膜的 EDS 图谱. 由图可见, 6.32 和 $6.87 \mathrm{keV}$ 的谱峰来自于 $\mathrm{Fe}, 7.43 \mathrm{keV}$ 的谱峰来自 $\mathrm{Ni}, 0.52 \mathrm{keV}$ 与 $2.31 \mathrm{keV}$ 的谱峰则分别来自于 $\mathrm{O}$ 和 $\mathrm{S}$ (与复合膜中 PANI 掺杂硫酸根离子有关), 说明复 合膜中同时具有 $\mathrm{NiHCF}$ 与 PANI 组分, 这与红外分 析所得的结果一致. 另外, $0.265 \mathrm{keV}$ 的谱峰源于 C, $1.47 \mathrm{keV}$ 的谱峰来自于 $\mathrm{Al}$ (制备 $\mathrm{CNTs}$ 的催化剂载 体 $\mathrm{Al}_{2} \mathrm{O}_{3}$ 的残留物), $2.05 \mathrm{keV}$ 的谱峰来自于 $\mathrm{Pt}$, 且三 者的谱峰远远大于 $\mathrm{Fe}$ 和 $\mathrm{Ni}$, 这主要由于复合膜是 在 $\mathrm{Pt} / \mathrm{CNTs}$ 修饰电极上制备的, 而 $\mathrm{K}$ 谱峰 $(3.29 \mathrm{keV})$ 的出现主要源于电解液中所带的 $\mathrm{K}$ 离子.

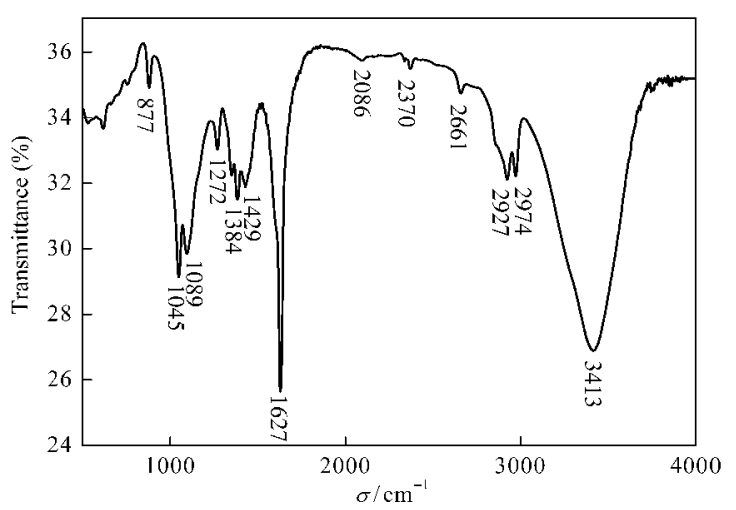

图 $3 \mathrm{CNTs} / \mathrm{PANI} / \mathrm{NiHCF}$ 复合膜的 FT-IR 谱图 Fig.3 FT-IR spectrum of CNTs/PANI/NiHCF film 


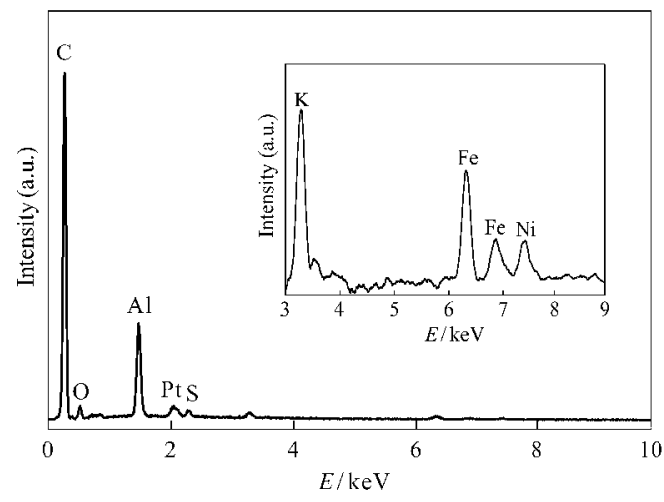

图 $4 \mathrm{CNTs} / \mathrm{PANI} / \mathrm{NiHCF}$ 复合膜的 EDS 图

Fig.4 EDS of CNTs/PANI/NiHCF film

The inset is an enlargement of the EDS between 3 and $9 \mathrm{keV}$.

图5为复合膜的扫描电子显微图像(SEM). 如图 所示, CNTs/PANI/NiHCF 复合膜呈现出三维多孔网 络状结构特征, PANI 和 NiHCF 由于静电吸引作用 形成立方体纳米复合颗粒, 尺寸均匀(约50-80 nm) 并呈串珠方式附着分布在 CNTs 表面. Kulesza 等 ${ }^{[3]}$ 在玻碳电极上制备的 PANI/NiHCF 复合膜由于没有 CNTs 基体, 其形貌和微结构与单一的 PANI 膜相 似, NiHCF 组分完全被 PANI 所分隔, NiHCF 的氧 化还原需通过相邻的 PANI 组分传递电荷才能进 行, 而 PANI 需要酸性溶液提供质子才能导电, 因而 这种复合膜在中性溶液中会失去电活性. Lin 等 ${ }^{[3]}$ 采 用两步电沉积技术合成的 CNTs/PANI/NIHCF 复合 膜在 CNTs 表面只存在 NiHCF 的不规则球形纳米 颗粒(粒径约 20-50 nm), PANI 和 NiHCF 先后分步 沉积并不能实现分子或晶格尺度的杂化. 而一步共 沉积 CNTs/PANI/NiHCF 复合膜呈现出完全不同的 形貌和微结构特征, 独特的三维多孔网络结构和以 纳米形式存在的 PANI/NiHCF 颗粒形成了大量的空

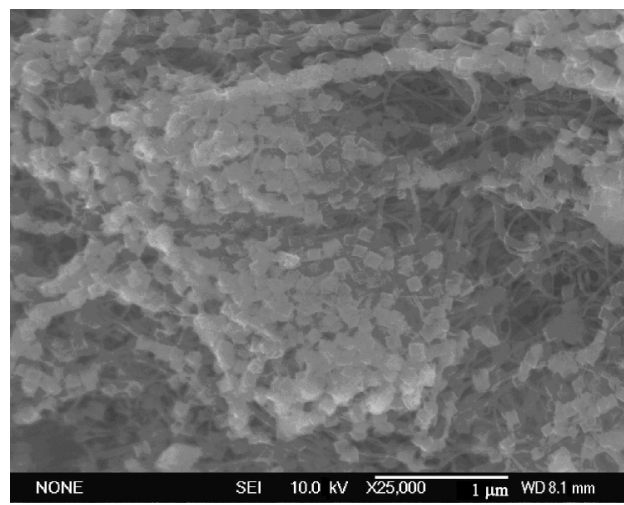

图 $5 \mathrm{CNTs} / \mathrm{PANI} / \mathrm{NiHCF}$ 复合膜的 SEM 照片 Fig.5 SEM image of CNTs/PANI/NiHCF film

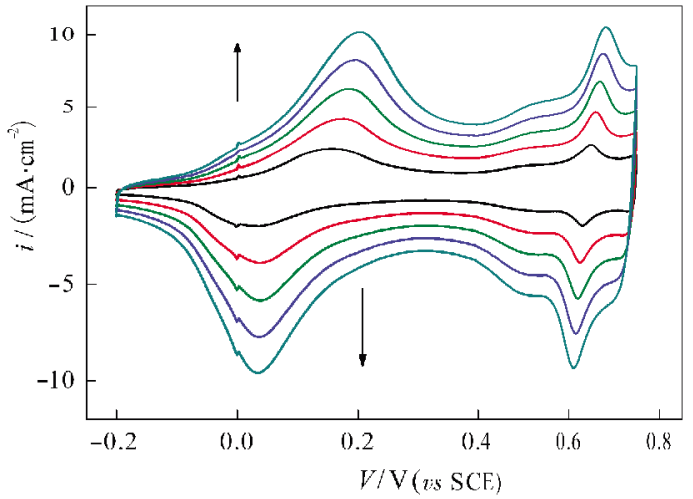

图 $6 \mathrm{CNTs} / \mathrm{PANI} / \mathrm{NiHCF}$ 复合膜在不同扫描速率下的 CV 曲线

Fig.6 CV curves of CNTs/PANI/NiHCF films at different scan rates

scan rate increase along with the arrow: $10,20,30,40,50 \mathrm{mV} \cdot \mathrm{s}^{-1}$

隙, 具有大的比表面积和优良的导电性, 从而可以减 小薄膜电极的极化现象和提高薄膜的比容量和比能 量, 并有利于提高膜内的离子传递速率和复合膜的 循环稳定性.

\section{3 循环伏安测试}

图 6 为 $\mathrm{CNTs} / \mathrm{PANI} / \mathrm{NiHCF}$ 复合膜在 $0.5 \mathrm{~mol}$. $\mathrm{L}^{-1} \mathrm{H}_{2} \mathrm{SO}_{4}+0.5 \mathrm{~mol} \cdot \mathrm{L}^{-1} \mathrm{KNO}_{3}$ 混合溶液中不同扫描速 度下 $\left(10,20,30,40,50 \mathrm{mV} \cdot \mathrm{s}^{-1}\right)$ 的 $\mathrm{CV}$ 曲线, 电位扫 描范围为 $-0.2-0.75 \mathrm{~V}$. 由图可见, 当扫描速度发生 改变时有快速的电流响应, 峰电位基本没有太大偏 移, 表明复合膜内阻较小, 充放电过程具有良好的动 力学可逆性 ${ }^{[32]}$. 图 7 为低氧化还原电位峰电流密度 随扫描速度的变化关系. 由图可知, 同一电位下对 应的氧化还原峰电流随扫描速度增加而线性增大, 说明复合膜表面和内部发生快速可逆的氧化还原反 应, 功率特性良好. 当扫描速度为 $10 \mathrm{mV} \cdot \mathrm{s}^{-1}$ 时, 根 据 $\mathrm{CV}$ 图计算得 $\mathrm{CNTs} / \mathrm{PANI} / \mathrm{NiHCF}$ 复合膜比容量为

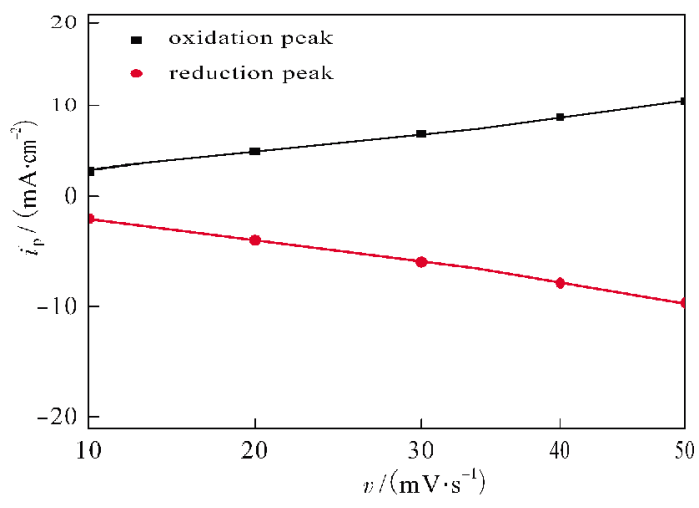

图 7 峰电流密度 $\left(i_{\mathrm{p}}\right)$ 与扫描速率 $(v)$ 的关系图

Fig.7 Plots of peak current density $\left(i_{\mathrm{p}}\right)$ versus scan rate $(v)$ 
$246.07 \mathrm{~F} \cdot \mathrm{g}^{-1}$, 而同样条件下 $\mathrm{CNTs} / \mathrm{PANI}$ 与 $\mathrm{CNTs} /$ $\mathrm{NiHCF}$ 的比容量分别为 195.56 和 $218.54 \mathrm{~F} \cdot \mathrm{g}^{-1}$. 随 着扫描速度(放电速率)的增加, 比容量发生一定程 度的衰减, 当扫描速度为 $50 \mathrm{mV} \cdot \mathrm{s}^{-1}$ 时, 复合膜比容 量为 $231.23 \mathrm{~F} \cdot \mathrm{g}^{-1}$, 衰减率仅为 $6.03 \%$. 说明了复合 膜具有快速的充放电速率和良好的稳定性, 是一种 较理想的电容器电极材料.

\section{4 恒电流充放电测试}

恒电流充放电是测试电极材料电容行为的重要 手段, 通常可藉放电曲线计算比电容大小. CNTs/ $\mathrm{PANI} / \mathrm{NiHCF}$ 复合膜在 $0.5 \mathrm{~mol} \cdot \mathrm{L}^{-1} \mathrm{H}_{2} \mathrm{SO}_{4}+0.5 \mathrm{~mol} \cdot \mathrm{L}^{-1}$ $\mathrm{KNO}_{3}$ 混合溶液中于不同电流密度下的充放电曲线 如图8所示. 由图可见, 所有电位-时间曲线基本呈 线性关系, 图形具有三角形对称分布, 说明复合膜在 不同电流密度下电化学可逆性和充放电性能良好, 表现出理想电容器的性能. 复合膜的比容量 $(C)$ 可通 过公式 $C=I \Delta t /(m \Delta E)$ 来计算. 式中 $\Delta E$ 为放电的电压 降, $\mathrm{V} ; \Delta t$ 为放电时间, $\mathrm{s} ; I$ 为放电电流, $\mathrm{A} ; m$ 为电极活 性物质质量, g. 根据上述公式计算出不同电流密度 下CNTs/PANI/NiHCF复合膜的比容量见表 1 .

由表 1 可知, 电流密度为 $2 \mathrm{~mA} \cdot \mathrm{cm}^{-2}$ 时, 复合膜 的比容量可达 $262.28 \mathrm{~F} \cdot \mathrm{g}^{-1}$. 随着电流密度的增加, 复合膜的比容量缓慢减少, 当电流密度为 $10 \mathrm{~mA}$ · $\mathrm{cm}^{-2}$ 时, 复合膜的比容量为 $256.61 \mathrm{~F} \cdot \mathrm{g}^{-1}$, 大电流下 充放电性能良好. 在大电流密度下, 比容量的降低主 要是由于在较短的时间内, 电解液中的离子或质子 来不及进人或离开电极表面, 使材料中的活性物质 没有得到有效利用, 比容量下降 ${ }^{[6}$. 而由一歩共聚法 制备的 CNTs/PANI/NiHCF 复合膜表面颗粒较小, 分布比较均匀, 使得活性物质间的电荷传递电阻较

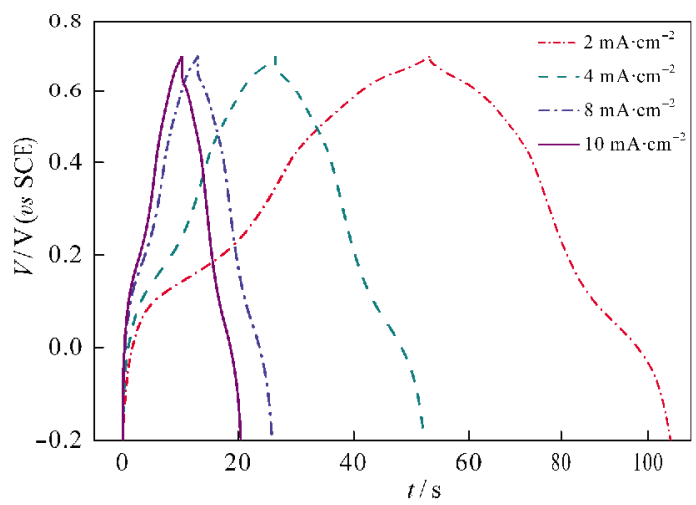

图 $8 \mathrm{CNTs} / \mathrm{PANI} / \mathrm{NiHCF}$ 复合膜在不同电流密度时 恒电流充放电曲线

Fig.8 Galvanostatic charge/discharge curves of CNTs/PANI/NiHCF films at different current densities
表 1 不同电流密度( $(i)$ 时 CNTs/PANI/NiHCF 复合膜的 比容量 $(\boldsymbol{C})$ 、比能量 $(\boldsymbol{W})$ 和比功率 $(\boldsymbol{P})$

Table 1 Specific capacitance $(C)$, specific energy

$(W)$, and specific power $(P)$ at differen current densities $(i)$ for CNTs/PANI/NiHCF films

\begin{tabular}{cccc}
\hline$i /\left(\mathrm{mA} \cdot \mathrm{cm}^{-2}\right)$ & $C /\left(\mathrm{F} \cdot \mathrm{g}^{-1}\right)$ & $W /\left(\mathrm{Wh} \cdot \mathrm{kg}^{-1}\right)$ & $P /\left(\mathrm{W} \cdot \mathrm{kg}^{-1}\right)$ \\
\hline 2 & 262.28 & 29.51 & 2045.87 \\
4 & 261.67 & 29.43 & 4091.16 \\
8 & 258.64 & 29.09 & 8183.37 \\
10 & 256.61 & 28.87 & 10228.61 \\
\hline
\end{tabular}

小, 有利于膜内离子和电子的扩散, 从而提高薄膜在 大电流密度下的充放电性能. 而且和电池相比, 超级 电容器的最大优点就是具有更高的比功率, 可以实 现大电流充放电而不影响其性能. 比能量 $(W)$ 和比功 率 $(P)$ 分别采用公式 $W=0.5 C \Delta E^{2}$ 和 $P=W / \Delta t$ 来计算. 复合膜的比能量可达 $29.51 \mathrm{Wh} \cdot \mathrm{kg}^{-1}$, 且随着电流密 度的增加基本不变, 同时具有较高的比功率 $\left(10228.61 \mathrm{~W} \cdot \mathrm{kg}^{-1}\right)$, 这是由于复合膜本身具有高而 稳定的比能量和较快的离子迁移速率, 使 $\mathrm{K}^{+}$和 $\mathrm{H}^{+}$在 薄膜中能够更加快速地嵌人与脱嵌.

\section{5 循环性能测试}

为进一步测试 $\mathrm{CNTs} / \mathrm{PANI} / \mathrm{NiHCF}$ 复合膜的循 环稳定性, 在电流密度为 $20 \mathrm{~mA} \cdot \mathrm{cm}^{-2}$ 时进行了 2000 次恒电流充放电测试. 由图 9 可知, 复合膜在 2000 次循环充放电下电容量仅衰减 $19.92 \%$, 电荷 充放电效率一直保持在 $99 \%$ 以上, 表现出稳定的电 化学容量性能. 同时在前 200 次循环过程中, 电容衰 减较快, 之后衰减速度变慢, 说明复合膜在快速充放 电过程中, 电化学反应不仅发生在电极表面, 而且存 在于复合膜所具有的三维网络结构之中, 随着循环 次数的增加, 离子或电子可快速进人或置出, 提高了

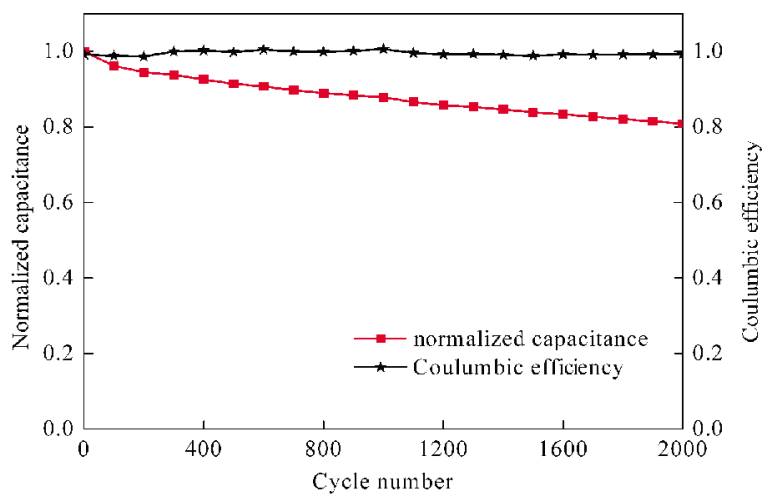

图 $920 \mathrm{~mA} \cdot \mathrm{cm}^{-2}$ 时 CNTs/PANI/NiHCF 复合膜的 循环性能图

Fig.9 Cycling performance of the CNTs/ANI/ NiHCF films at current density of $20 \mathrm{~mA} \cdot \mathrm{cm}^{-2}$ 


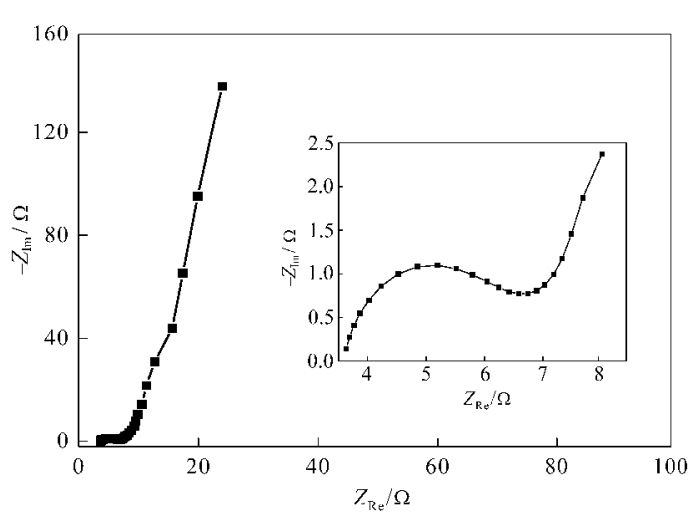

图 $10 \mathrm{CNTs} / \mathrm{PANI} / \mathrm{NiHCF}$ 复合膜的 EIS 图 Fig.10 EIS spectra of CNTs/PANI/NiHCF films

$E=0 \mathrm{~V}$, frequency range: $0.01 \mathrm{~Hz}-100 \mathrm{kHz}$, amplitude: $10 \mathrm{mV}$

材料的利用率, 循环慢慢趋于稳定. Zhang 等 ${ }^{[5}$ 报道 电化学聚合单一 CNTs/PANI 复合膜经过 1000 次充 放电循环比电容衰减量达 $32 \%$, Lisowska-Oleksiak 等 ${ }^{300}$ 通过两步电聚合制备的 PEDOT/NiHCF 有机无机杂化膜循环 500 次电容损失 $15 \%$, Lin 等 ${ }^{[35]}$ 通 过两步电沉积制得的 CNTs/PANI/NiHCF 复合膜经 500 次循环其离子交换电量也衰减 $8 \%$. 本实验采用 循环伏安一步共聚制备复合膜, 通过调节电势扫描 速度和电解液组成控制逐层沉积 PANI 和 NiHCF, 利用带正电荷的 PANI 与带负电荷的 NiHCF 之间 的静电吸引作用达到分子或晶格尺度的有机-无机 组分杂化, 在三维多孔网络结构的 CNTs 表面生成 复合纳米颗粒并产生协同效应, 因而具有优良的循 环稳定性能.

\section{6 电化学阻抗测试}

图 10 为 $\mathrm{CNTs} / \mathrm{PANI} / \mathrm{NiHCF}$ 复合膜的电化学 阻抗图谱, 测试电压 $0 \mathrm{~V}$, 正弦波幅值 $10 \mathrm{mV}$, 频率 范围 $0.1 \mathrm{~Hz}-100 \mathrm{kHz}$. 图谱中高频区的半圆表示电 极材料界面上的电荷转移电阻, 低频区的直线表示 与电化学容量相关的充电机理, 直线越垂直于横坐 标, 表明其电容性能越好 ${ }^{[3]}$. 由图可见, 低频段的直 线部分与横坐标接近垂直, 表现出理想电容器的特 征. 根据图 10 阻抗图谱的等效电路可求得复合膜溶 液电阻 $R_{\mathrm{s}}$ 约 $3.6 \Omega$, 电荷转移电阻 $R_{\mathrm{ct}}$ 约 $4.3 \Omega$. 考虑 到复合膜具有一定的厚度, $R_{\mathrm{s}}$ 与 $R_{\mathrm{ct}}$ 都是较小的, 表 明复合膜具有较低的电化学电阻和优良的电化学电 容行为, 这与图 6 所示循环伏安曲线所得的结果相 一致.

\section{3 结 论}

(1) 采用循环伏安一步共聚法可制得三维多孔
网络结构的 CNTs/PANI/NiHCF 复合膜, 有机-无机 杂化材料中带正电荷的 PANI 与带负电荷的 NiHCF 之间可通过静电吸引相互作用, 形成尺寸均一的立 方体纳米颗粒并沿 CNTs 均匀分布. 三维多孔结构 纳米复合膜将 CNTs 的高导电性和导电聚合物以及 过渡金属铁氧化物可逆氧化还原产生的㕍电容特性 相结合, 具有大的比表面积、低的离子扩散阻力和良 好的化学稳定性, 为超级电容器电极材料提供了高 的比电容性能.

(2) CNTs/PANI/NiHCF 复合膜在酸性和中性 电解液中均具有良好的电化学性能. 在 $0.5 \mathrm{~mol} \cdot \mathrm{L}^{-1}$ $\mathrm{H}_{2} \mathrm{SO}_{4}+0.5 \mathrm{~mol} \cdot \mathrm{L}^{-1} \mathrm{KNO}_{3}$ 混合溶液中当扫描速度从 $10 \mathrm{mV} \cdot \mathrm{s}^{-1}$ 增加到 $50 \mathrm{mV} \cdot \mathrm{s}^{-1}$ 时比容量衰减率为 $6.03 \%$; 比容量、比能量和比功率分别达到 $262.28 \mathrm{~F}$. $\mathrm{g}^{-1} 、 29.51 \mathrm{Wh} \cdot \mathrm{kg}^{-1}$ 和 $10228.61 \mathrm{~W} \cdot \mathrm{kg}^{-1}$; 在 2000 次充 放电循环中复合膜的电容量维持在 $80 \%$ 以上, 而电 荷充放电效率一直保持在 $99 \%$ 以上, 表现出稳定的 循环性能. 阻抗分析发现, 复合膜溶液电阻 $R_{\mathrm{s}}$ 约 3.6 $\Omega$, 电荷转移电阻 $R_{\mathrm{ct}}$ 约 $4.3 \Omega$, 表现出理想电容器的 特征.

(3) 电化学一步共聚 CNTs/PANI/NiHCF 复合膜 是一种优异的超级电容器电极材料, 在电催化、生物 传感器和新型电控离子交换(ESIX)等领域也有潜在 的应用前景.

\section{References}

1 Cottineau, T.; Toupin, M.; Delahaye, T.; Brousse, T.; Bélanger, D. J. Appl. Phys. A, 2006, 82: 599

2 Reddy, R. N.; Reddy, R. G. J. Power Sources, 2003, 124: 330

3 Du, B.; Jiang, Q.; Zhao, X. F.; Lin, S. Z.; Mu, P. S.; Zhao, Y. Acta Phys. -Chim. Sin., 2009, 25: 513 [杜＼cjkstart冰, 江 奇, 赵晓峰, 林孙 忠, 幕佩珊, 赵 勇. 物理化学学报, 2009, 25: 513]

4 Zhao, G. Y.; Li, H. L. Microporous Mesoporous Mat., 2008, 110: 590

5 Zhang, J.; Kong, L. B.; Wang, B.; Luo, Y. C.; Kang, L. Synth. Met., 2009, 159: 260

6 Chen, J.; Huang, K. L.; Liu, S. Q. Chin. J. Inorg. Chem., 2008, 24 : 621 [陈 洁, 黄可龙, 刘素琴. 无机化学学报, 2008, 24: 621]

7 Frackowiak, E.; Jurewicz, K.; Delpeux, S.; Beguin, F. J. Power Sources, 2001, 97-98: 822

8 Deng, M. G.; Yang, B. C.; Hu, Y. D.; Wang, B. H. Acta Chim. Sin., 2005, 63: 1127 [邓梅根, 杨邦朝, 胡永达, 汪斌华. 化学学报, 2005, 63: 1127]

9 Koysuren, O.; Du, C.; Pan, N.; Bayram, G. J. Appl. Polym. Sci., 2009, 113: 1070

10 Yang, H. S.; Zhou, X.; Zhang, Q. W. Acta Phys. -Chim. Sin., 2005, 
21: 414 [杨红生, 周 啸, 张庆武. 物理化学学报, 2005, 21: 414]

11 Li, W. K.; Chen, J.; Zhao, J. J.; Zhang, J. R.; Zhu, J. J. Mater. Lett., 2005, 59: 800

12 Lai, Y. Q.; Lu, H.; Zhang, Z. A.; Li, J.; Li, J.; Liu, Y. X. J. Cent. South Univ., 2007, 38: 1110 [赖延清, 卢 海, 张治安, 李 晶, 李 款, 刘业翔. 中南大学学报, 2007, 38: 1110]

13 Wang, J.; Xu, Y. L.; Sun, X. F.; Xiao, F.; Mao, S. C. Acta Phys. Chim. Sin., 2007, 23: 877 [王 杰, 徐友龙, 孙孝飞, 肖 芳, 毛 胜春. 物理化学学报, 2007, 23: 877]

14 Mi, H.; Zhang, X.; Yang, S.; Ye, X.; Luo, J. Mater. Chem. Phys., 2008, 112: 127

15 Palaniappan, S.; Devi, S. L. J. Appl. Polym. Sci., 2008, 107: 1887

16 Liu, F. J. J. Power Sources, 2008, 182: 383

17 Skunik, M.; Chojak, M.; Rutkowska, I. A.; Kulesza, P. J. Electrochim. Acta, 2008, 53: 3862

18 Zelikman, E.; Narkis, M.; Siegmann, A.; Valentini, L.; Kenny, J. M. Polym. Eng. Sci., 2008, 48: 1872

19 Hu, Z. A.; Xie, Y. L.; Wang, Y. X.; Mo, L. P.; Yang, Y. Y.; Zhang, Z. Y. Mater. Chem. Phys., 2009, 114: 990

20 Wang, J.; Xu, Y. L.; Chen, X.; Du, X. F.; Li, X. F. Acta Phys. Chim. Sin., 2007, 23: 299 [王 杰, 徐友龙, 陈 䂀, 杜显锋, 李喜飞. 物理化学学报, 2007, 23: 299]

21 Wang, J.; Xu, Y.; Chen, X.; Sun, X. Compos. Sci. Technol., 2007, 67: 2981

22 Hao, X. G.; Schwartz, D. T. Chem. Mater., 2005, 17: 5831

23 Hao, X. G.; Guo, J. X.; Liu, S. B.; Sun, Y. P. Trans. Nonferrous Met. Soc. China, 2006, 16: 556
24 Hao, X. G.; Li, Y. G.; Pritzker, M. Sep. Puri. Technol., 2008, 63: 407

25 Chen, W.; Xia, X. H. Adv. Funct. Mater., 2007, 17: 2943

26 Chen, J.; Huang, K. L.; Liu, S. Q. Electrochem. Commun., 2008, 10: 1851

27 Shahrokhian, S.; Ghalkhani, M.; Adeli, M.; Amini, M. K. Biosens. Bioelectron., 2009, 24: 3235

28 Qiu, J. D.; Xiong, M.; Liang, R. P.; Zhang, J.; Xia, X. H. J. Nanosci. Nanotechnol., 2008, 8: 4453

29 Lisowska-Oleksiak, A.; Nowak, A. P. Solid State Ionics, 2008, 179: 72

30 Lisowska-Oleksiak, A.; Nowak, A. P. J. Power Sources, 2007, 173: 829

31 Makowski, O.; Kowalewska, B.; Szymanska, D.; Stroka, J.; Miecznikowski, K.; Palys, B.; Malik, M. A.; Kulesza, P. J. Electrochim. Acta, 2007, 53: 1235

32 Kulesza, P. J.; Skunik, M.; Baranowska, B.; Miecznikowski, K.; Chojak, M.; Karnicka, K.; Frackowiak, E.; Beguin, F.; Kuhn, A.; Delville, M.; Starobrzynska, B.; Ernst, A. Electrochim. Acta, 2006, 51: 2373

33 Skunik, M.; Kulesza, P. J. Anal. Acta Chim., 2009, 631: 153

34 Kulesza, P. J.; Miecznikowski, K.; Malik, M. A.; Galkowski, M.; Chojak, M.; Caban, K.; Wieckowski, A. Electrochim. Acta, 2001, 46: 4065

35 Lin, Y.; Cui, X. J. Mater. Chem., 2006, 16: 585

36 Zhan, S.; Tian, Y.; Cui, Y.; Wu, H.; Wang, Y.; Ye, S.; Chen, Y. China Particuology, 2007, 5: 213

37 Pile, D. L.; Hillier, A. C. J. Membr. Sci., 2002, 208: 119 